Supporting information

\title{
pH-Sensitive Carbon Dots for Enhancing Photomediated Antitumor Immunity
}

Da hye Kim, Jeongdeok Seo, Kun Na*

Department of Biotechnology, Department of Biomedical-Chemical Engineering, The Catholic University of Korea,

43 Jibong-ro, Wonmi-gu, Bucheon-si, Gyeonggi-do, 14662, Republic of Korea

*E-mail: kna6997@catholic.ac.kr 
(a)

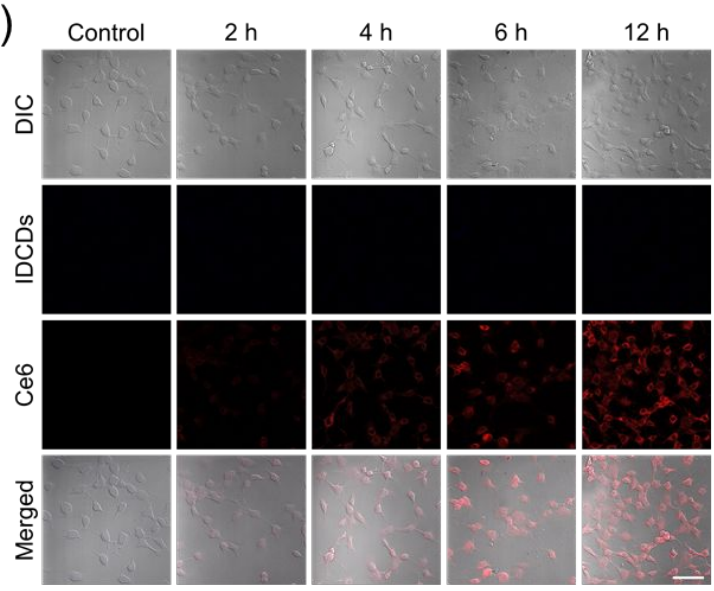

(c)

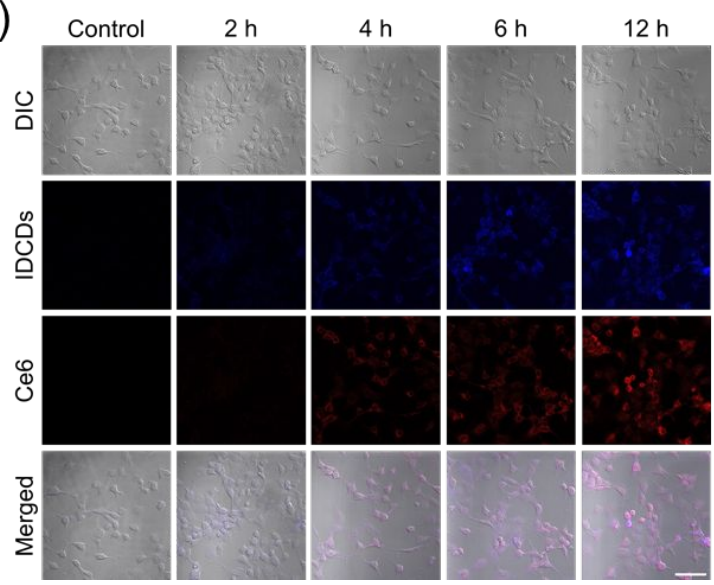

(b)

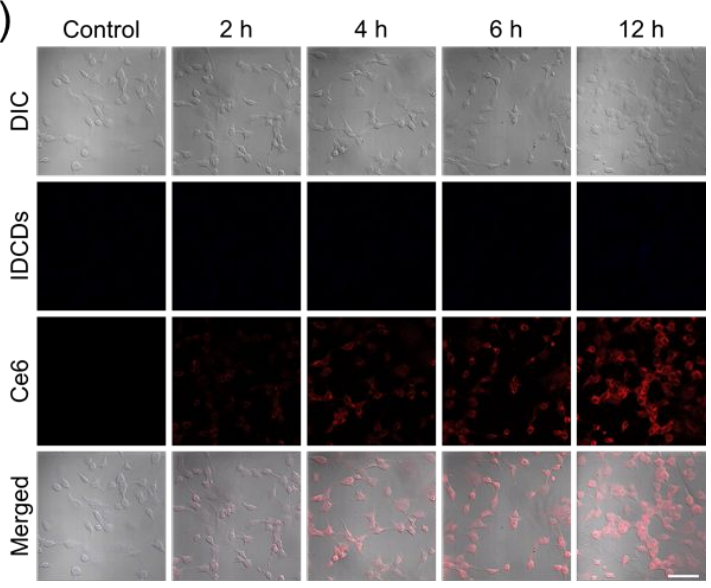

(d)

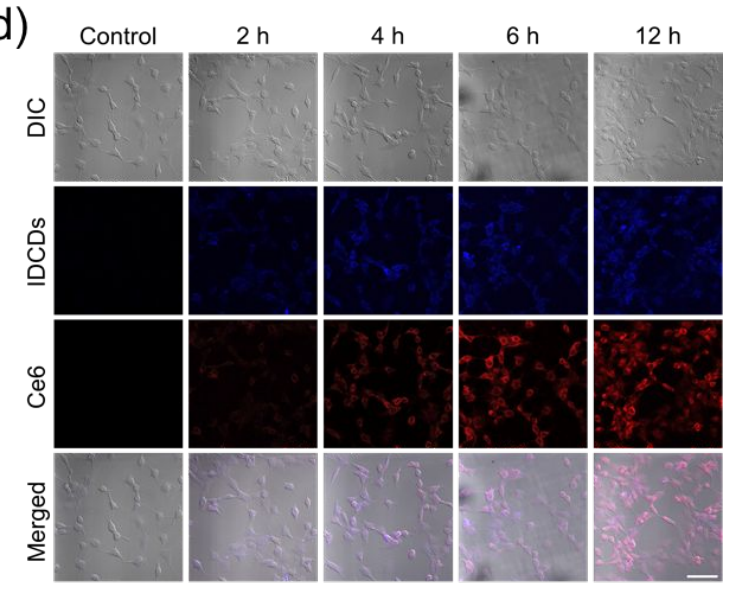

Figure S1. CLSM images of intracellular distribution of Free Ce6 at (a) pH 7.4 or (b) pH 6.5 and Ce6@IDCDs at (c) pH 7.4 or (d) pH 6.5 (dose of Ce6; $1 \mu \mathrm{g} / \mathrm{mL}$ ) in CT-26 cells for predetermined duration. Scale bar, $100 \mu \mathrm{m}$. 


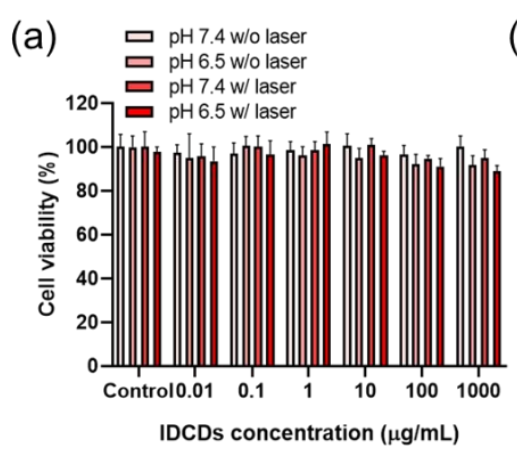

(b)

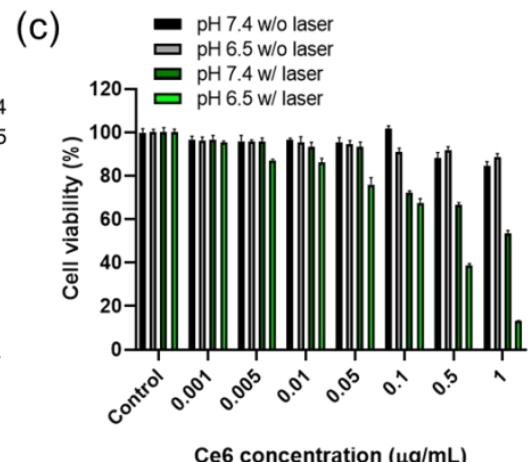

Figure S2. The cytotoxicity of (a) IDCDs with laser irradiation, (b) Free Ce6 without laser irradiation and (c) Ce6@IDCDs with laser irradiation dependent on various pH against CT-26 cells for $4 \mathrm{~h}\left(\mathrm{n}=4,0.05 \mathrm{~W} / \mathrm{cm}^{2}\right.$ for $\left.40 \mathrm{~s}\right)$. 
(a)

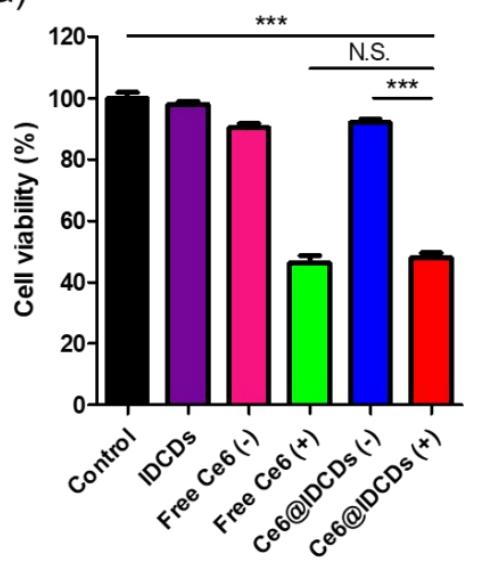

(b) Ce6@IDCDs Ce6@IDCDs

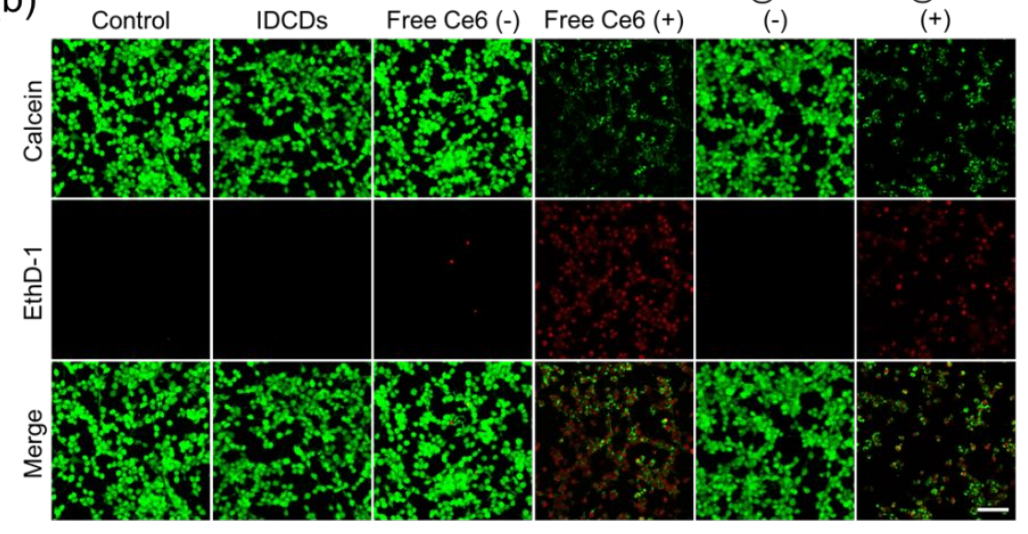

Figure S3. In vitro phototoxicity of Ce6@IDCDs in CT-26 cells at pH 7.4. (a) MTT colorimetric assay $(\mathrm{n}=4) .{ }^{* * *} P<0.001$, N.S., not significant (one-way ANOVA with Tukey post hoc analysis). (b) Live/dead assay of CT-26 cells incubated with each sample (dose of Ce6; $1 \mu \mathrm{g} / \mathrm{mL}$, dose of IDCDs; $12.5 \mu \mathrm{g} / \mathrm{mL}$ ) for $4 \mathrm{~h}$ at normal $\mathrm{pH} 7.4$ upon laser irradiation $\left(0.05 \mathrm{~W} / \mathrm{cm}^{2}\right)$ for $40 \mathrm{~s}$. 
(a)

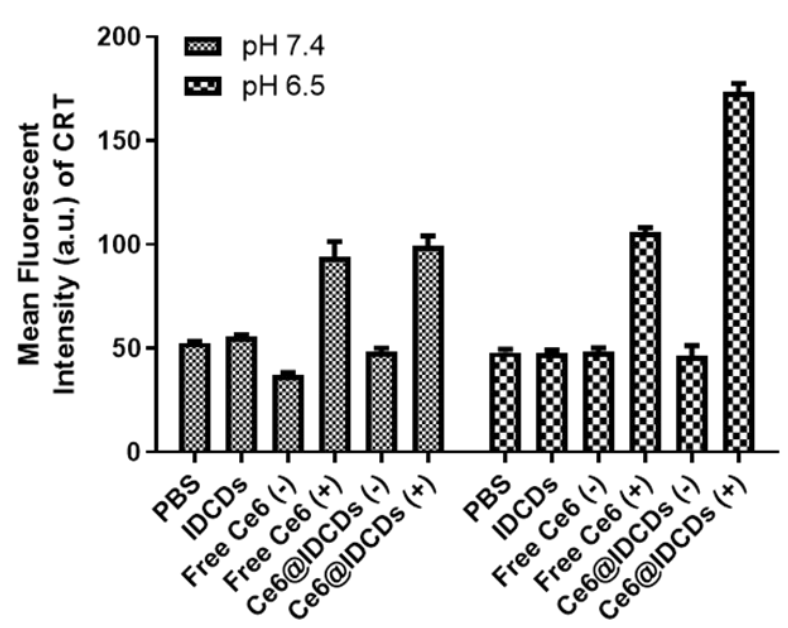

(b)
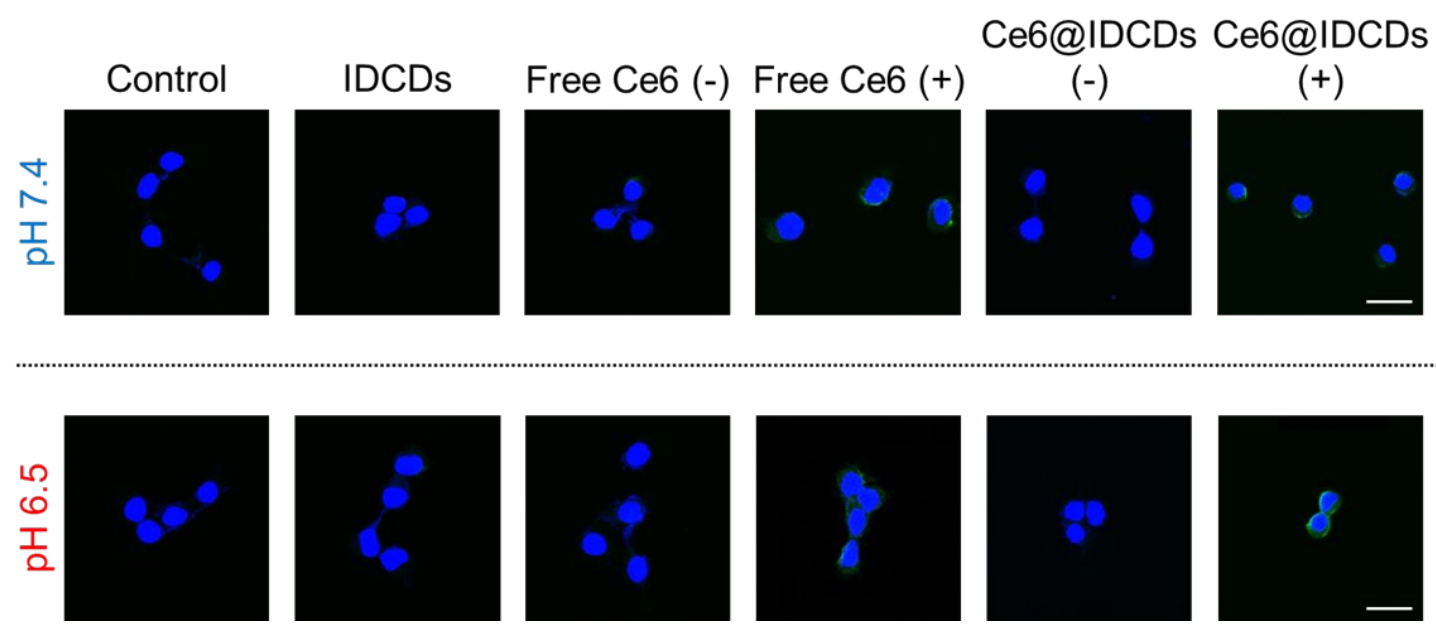

Figure S4. Comparison of calreticulin exposure level in CT-26 cells 2 hours after treated with different formulations treatment at $\mathrm{pH} 7.4$ or 6.5 upon laser irradiation analyzed by (a) flow cytometry and (b) CLSM images (Scale bars, $20 \mu \mathrm{m}$ ). 
(a)

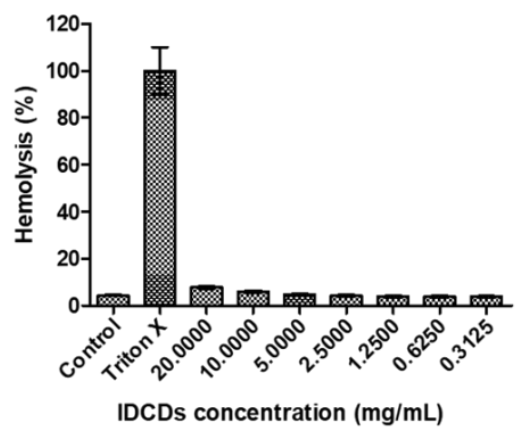

(b)

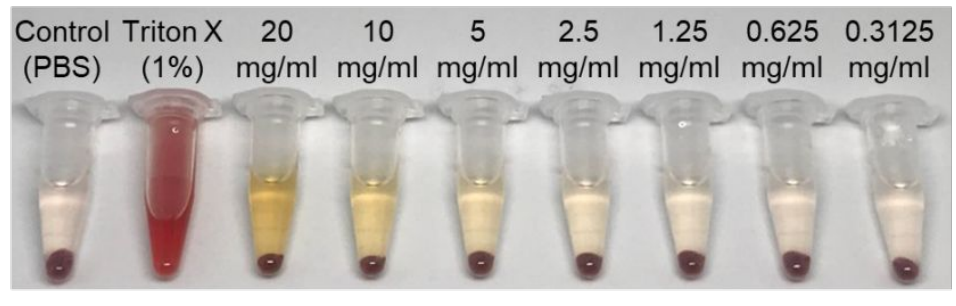

Figure S5. Hemolysis assay of IDCDs. (a) Hemolytic percentage $(n=5)$ and (b) photograph of red blood cells after treatment of various concentration of IDCDs. 1\% Triton X was used as a positive control (100\% hemolysis). 
(a)

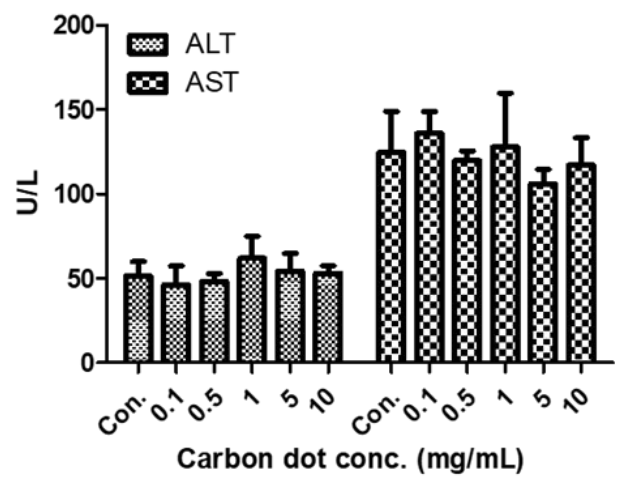

(b)

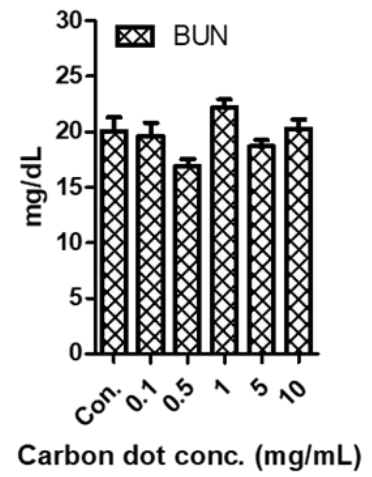

(c)

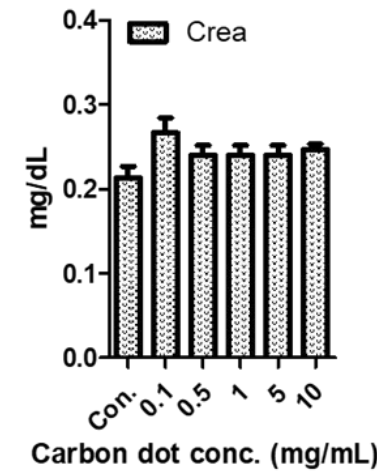

Figure S6. Biochemical and liver/kidney function assessment. Effects of IDCDs on (a) alanine aminotransferase (ALT) and aspartate aminotransferase (AST), (b) blood urea nitrogen (BUN), and (c) creatinine (Crea) levels in serum. Data are shown as mean $\pm \mathrm{SD}(\mathrm{n}=5)$. 


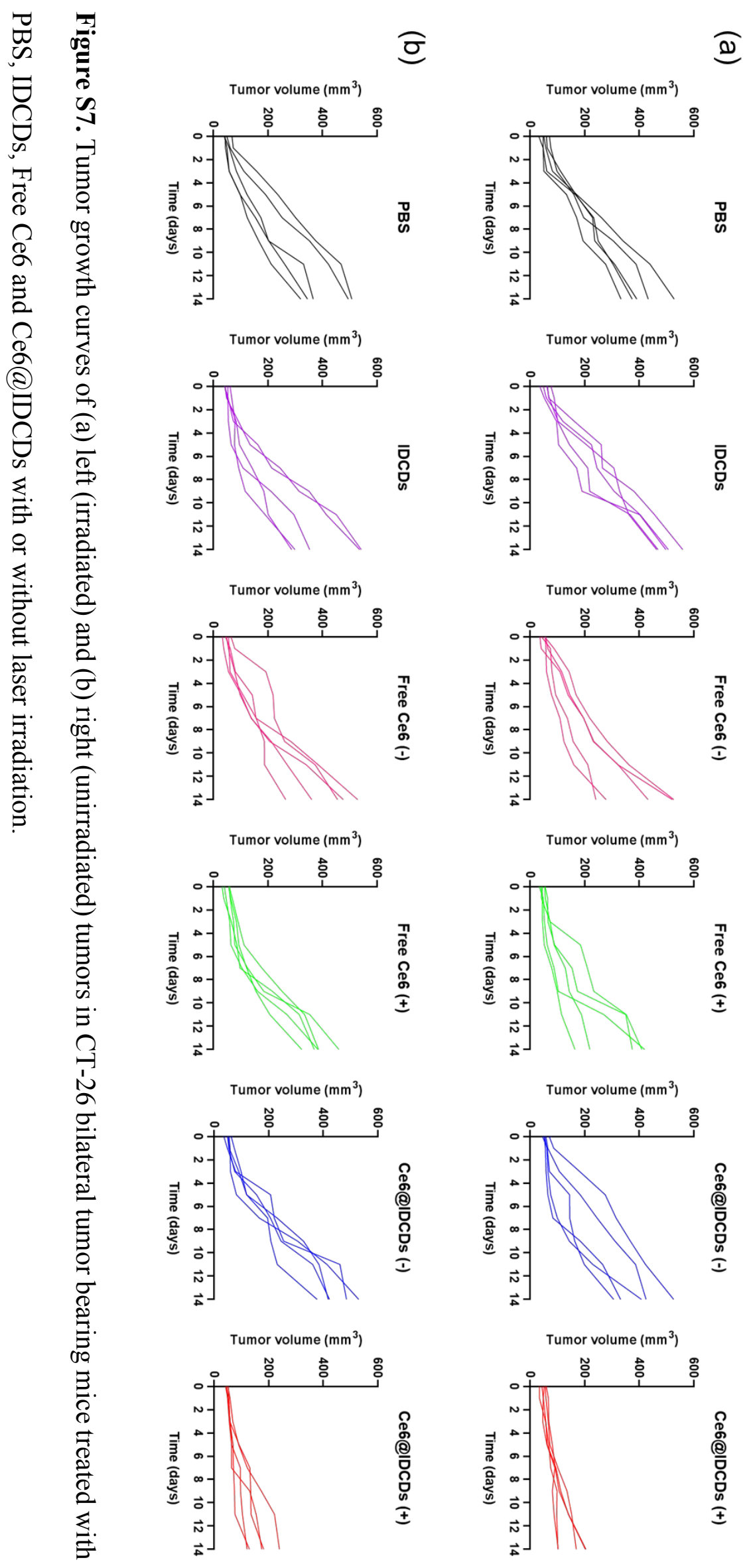




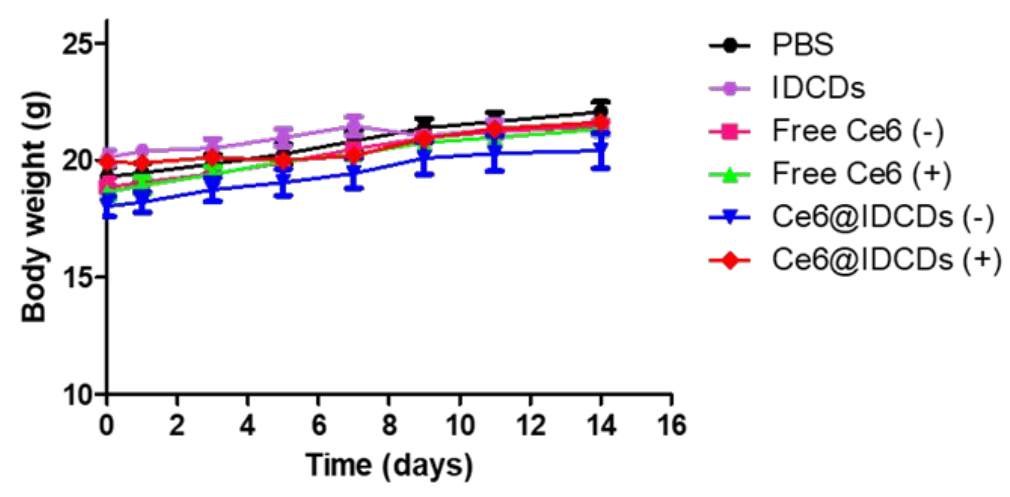

Figure S8. Body weight measurement of CT-26 bilateral tumor-bearing mice for 14 days. 


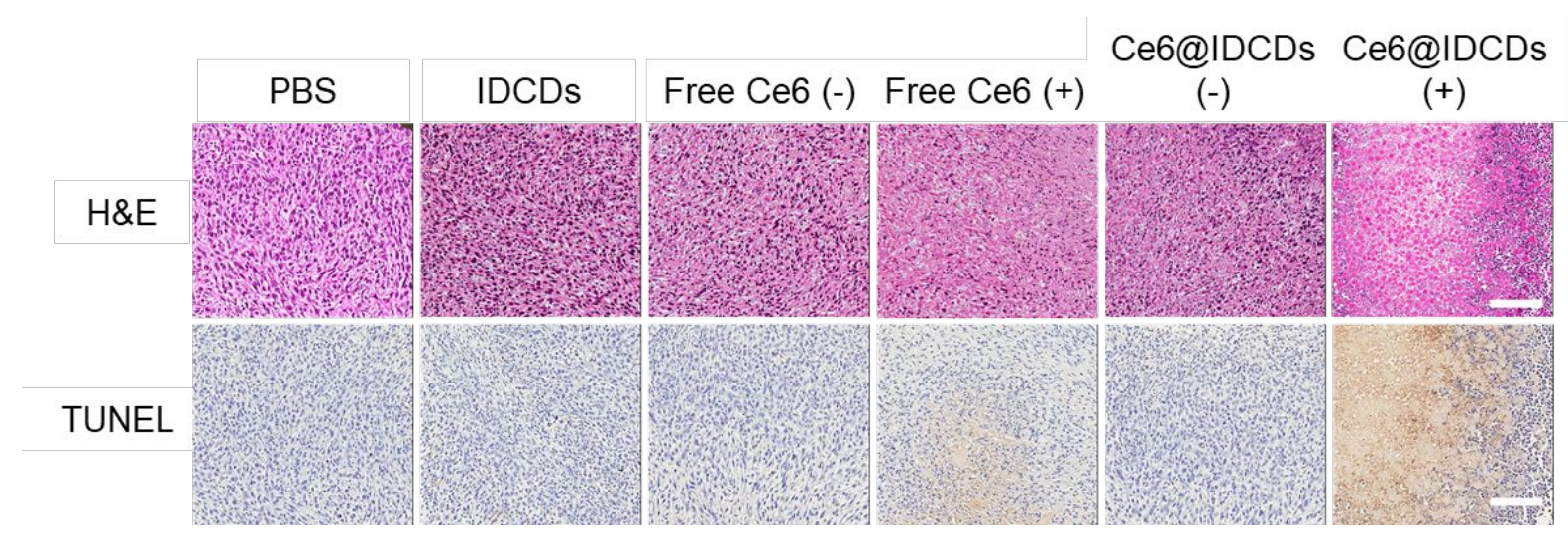

Figure S9. Histological observation of tumor tissues stained with H\&E and TUNEL staining after 14 days. Nuclei were stained blue, and the extracellular matrix and cytoplasm were stained red in H\&E staining. Brown color indicates apoptotic cells after a TUNEL assay. Scale bars, $100 \mu \mathrm{m}$. 


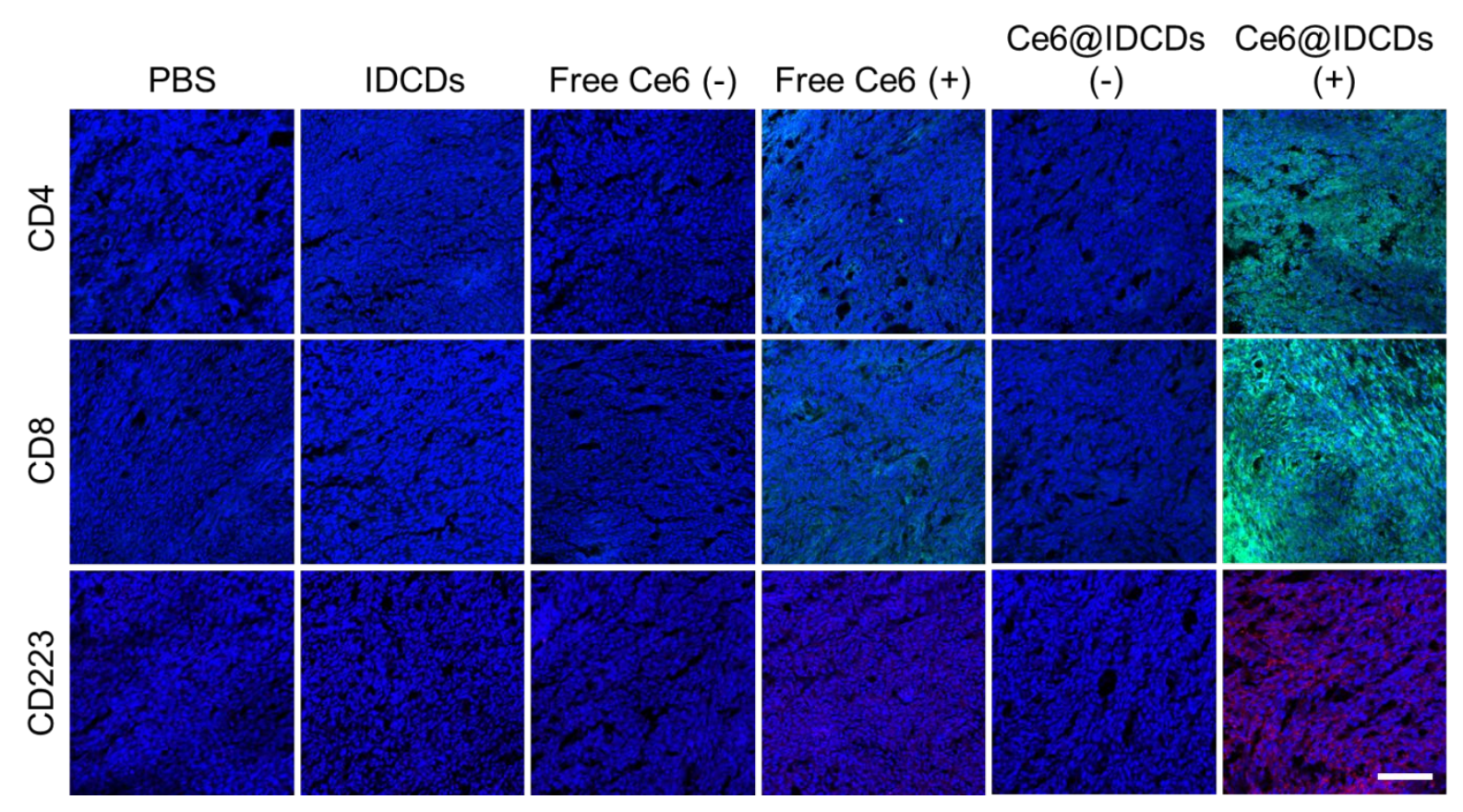

Figure S10. In vivo recruited immune cells $\left(\mathrm{CD}^{+}, \mathrm{CD} 8^{+} \mathrm{T}, \mathrm{NK}\right.$ cells $)$ in tumor tissues from PBS, IDCDs, Free Ce6 or Ce6@IDCDs at day 14 (dose of Ce6; 2 mg/kg) injected CT-26 tumor-bearing mice with or without laser irradiation using $671 \mathrm{~nm}$ light $(\mathrm{n}=5)$. (Scale bar $=$ $100 \mu \mathrm{m})$. 